\title{
Fasts Laser POF side Long Period Gratings fabrication
}

\author{
H.S. G. Roufael ${ }^{1}$, J. Castrellon-Uribe ${ }^{1,2}$, M. Lomer ${ }^{1^{*}}$, and J.M. Lopez-Higuera ${ }^{1}$ \\ ${ }^{1}$ Photonics Engineering Group, Cantabria University, Av. De los Castros s.n. 39005 Santander, Spain \\ ${ }^{2}$ Centro de Investigación en Ingeniería y Ciencias Aplicadas, CIICAp, Universidad Autónoma del \\ Estado de Morelos, UAEM, Av. Universidad 1001, Col. Chamilpa, Cuernavaca Morelos, México. \\ *lomerm@unican.es
}

\begin{abstract}
By using a fiber laser chirp pulse amplifier femtosecond laser system, long period gratings structures are micro-machined on one side of a Plastic Optical Fiber. Very narrow and high attenuation transmission bands can be obtained.
\end{abstract}

OCIS codes: (060.2340) Fiber optics components; (060.2290) Fiber materials

\section{Introduction}

Long Period Grating (LPG) are periodic optical structures what period is many times higher than the used interrogation wavelengths. LPG's on fibers are useful optical structures able to couple the core mode/s to the cladding modes under the phase match condition [1]. Then a series of notches at specific wavelengths (resonances ones) of the transmission spectrum are obtained. Currently the periodic LPG pattern is inscribed in the core of the optical fibers. However, it could also be inscribed in the cladding and then very sensible fiber structures to the surrounded fiber ambient measurands can be obtained [2]. In this paper, very accurate patters are photo-inscribed on one side of a step-index polymethyl-methacrylate (PMMA) Plastic Optical Fibers (POF) using a fiber based femtosecond laser. Notches in near infrared and inside the visible bands of the transmission spectrum can be easily obtained. The experimental set up, samples of the fabricated LPGs and some of the experimental results are reported in the next sections.

\section{Experimental Setup}

An illustration of the experimental set-up used is shown in figure 1. The fast source output beam is properly guided and steered to the focusing high numerical aperture objective. The later output is focused onto the side of the POF fiber placed on and ultrahigh resolution XYZ computer controlled stage. The fabrication process is online monitored by launching a white light source into the core fiber and the transmission spectra is recorded by using an Optical Spectrum Analizer (OSA), computer controlled.

The fast source is a fiber laser chirp pulse amplifier (FLCPA) from CALMAR lasers (CAZADERO) that are able to produce pulses of $360 \mathrm{fs}$ at $1030 \mathrm{~nm}$ and with an energy of 20 micro-joules. To guarantee the maximum stability during the micro-fabrication process, high stable guiding mirrors, objective focal lens, and other components have been fixed on a customized metallic construction rails which has been built and fixed over a highly stable optical table with vibrations isolation system. To have a live view to the micro-machined line by line a CCD camera

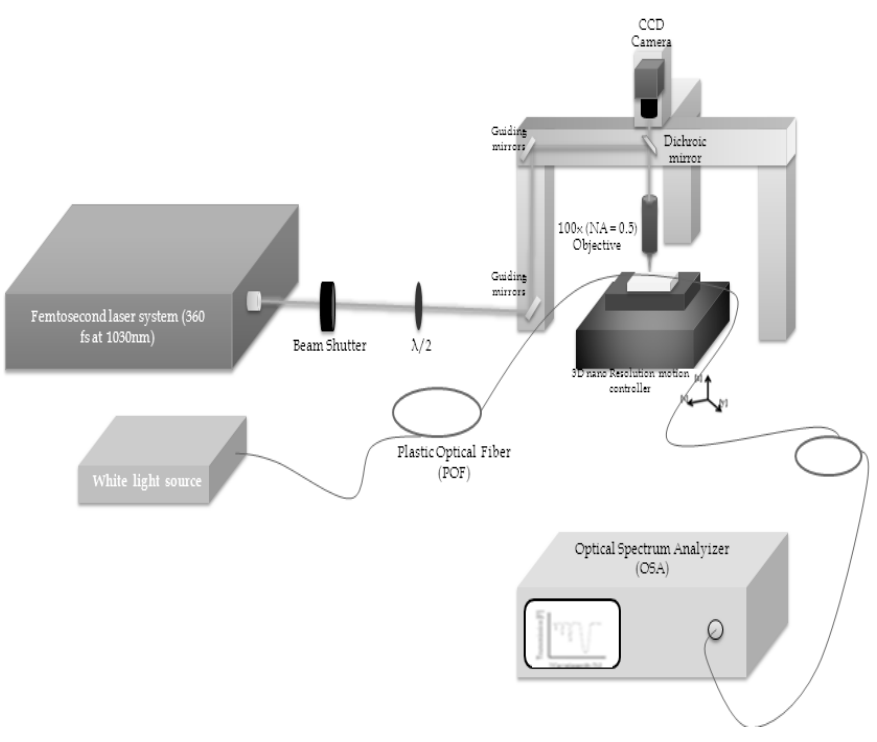
is fixed aver the dichroic mirror (80:20). Fig.1.- General illustration of the experimental setup.

The POF fiber used is a SK10 step index fiber what main characteristics are as follow: $240 / 250 \mu \mathrm{m}$ core/clad fiber diameters; PMMA polymer core; fluorinated polymer cladding; numerical aperture: 0.5 ; core refractive index $=1.49$ at $600 \mathrm{~nm}$; and 1.484 at $800 \mathrm{~nm}$. Cladding refractive index $=1.40$. 


\section{Experimental results}

A schematic view of the lateral laser micro-machined LPG grating structure is illustrated on the left hand of the Figure 2. On the right hand, details of one of the fabricated structures are shown.
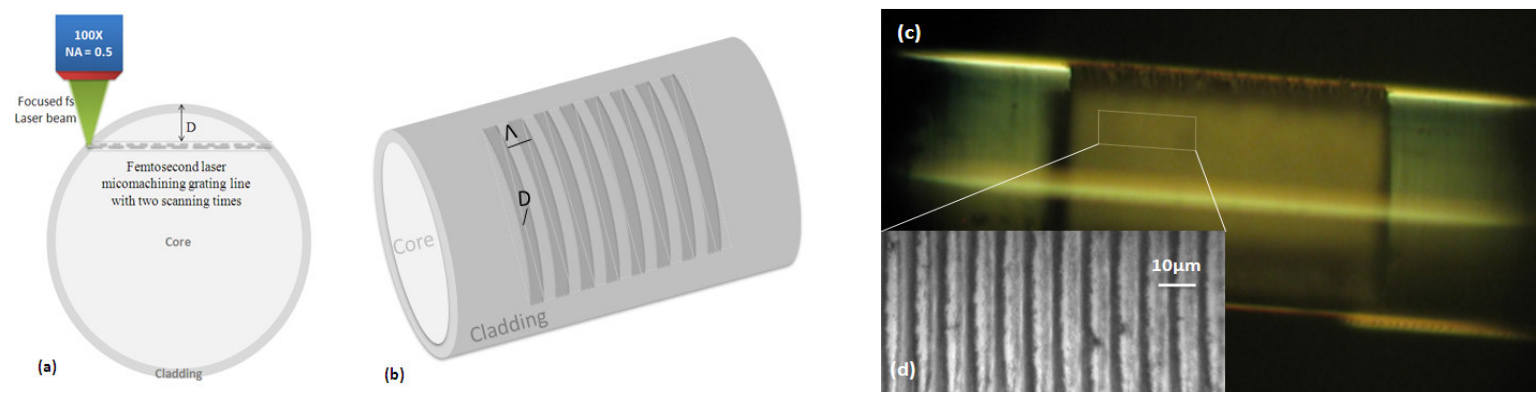

Fig.2 (a) An Illustration of the laser micromachining LPG, (b) representing a general view of the micromachining gratings shape, where $\Lambda$ is the grating period and D is the micro-machined line depth, (c) A general view for the experimental grating results in POF, and (d) Microscopic image for the laser micro-machined grating lines on the POF surface with a $50, \Lambda=9 \mu \mathrm{m}$, depth of about $25 \mu \mathrm{m}$.

A wide number of structures with different periods and different grating micro-machined depths were fabricated and characterized. In figure 3 results from two samples are shown.
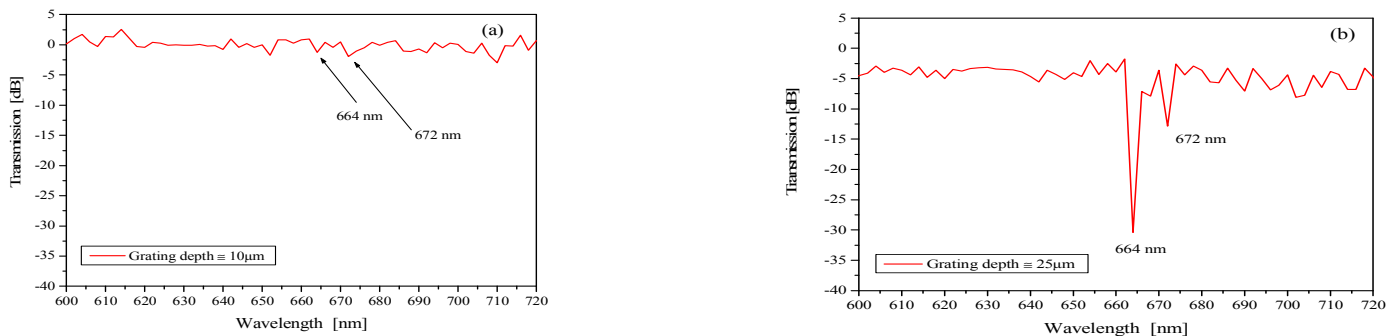

Fig.3.- Experimental transmission results for the fabricated LPG in POF (50 lines of $\Lambda=9 \mu \mathrm{m}$ of period) with different line depths for two transmission bands notches at $664 \mathrm{~nm}$, and $672 \mathrm{~nm}$. (a) Micro-machined LPG lines with very low depth and (b) Micro-machined LPG lines with higher depth $(25 \mu \mathrm{m})$. Laser: $\lambda=1030 \mathrm{~nm} ; \tau=360 \mathrm{fs}$; pulse energy $=2.4$ micro Joules.

\section{Conclusions}

By using a fiber femtosecond laser system, LPG structures are fabricated in one side of a SK10 step-index PMMAPOF. With the point-by point method, high precision and complex structures are easily fabricated. By managing the laser pulse energy, the pulse repetition rate and XYZ displacement speeds, LPG's transmission band notches with custom properties can be obtained. As high as $25 \mathrm{dBs}$ rejection bands can be easily fabricated inside the visible spectrum with the penalty of higher LPG insertion losses. The higher the rejection peak the higher the insertion loss. This special side POF-LPG structure (bio-compatible) can be used as transducer for surrounding ambient sensing for measurands such as: refraction indexes, temperature, strain and, with special patterns, torsion [3].

Acknowledgement: this work was supported by the MCYT (Spain), project CICYT: TEC2010-20224-C02-02 and CONACyT (Mexico) by sabbatical stay at the University of Cantabria, file: 186243.

\section{References}

[1] A. M. Vengsarkar, P. J. Lemaire, J. B. Judkins, V. Bhatia,T. Erdogan. JLT, 14, 58 (1996).

[2] JM López-Higuera, Editor, " Handbook of Optical Fibre Sensing Technology", John Wiley \& Sons Inc., ISBN 0-47182-053-9. 2002

[3] Tao Zhu, Leiley Shi, Min Liu, and Wei Huang; Journal of sensors, Hindawi Publ. Corpor., Vol, 2012 article ID 539095. 